\title{
EVERY UNIFORMLY CONTINUOUS CENTERED SEMIGROUP IS NORMAL
}

\author{
MARY EMBRY - WARDROP
}

\begin{abstract}
A well-known result on commutators of operators is used to prove the assertion in the title.
\end{abstract}

Consider a strongly continuous semigroup $\left(S_{t}: t \geqslant 0\right)$ of continuous linear operators on a Hilbert space $\mathscr{H}$. Let $U_{t} P_{t}$ be the canonical polar factorization of $S_{t}$. The semigroup $\left(S_{t}\right)$ is said to be centered if the partially isometric factor $\left(U_{t}\right)$ of $\left(S_{t}\right)$ is a semigroup. Examples of strongly continuous nonnormal centered semigroups are given in [1]. The purpose of this note is to demonstrate the truth of the assertion given in the title.

THEOREM. Every uniformly continuous centered semigroup is normal.

Proof. Let $\left(S_{t}\right)$ be a uniformly continuous centered semigroup with (bounded) generator $A$; that is, $S_{t}=e^{t A}$. We need only show that the operator $A$ is normal and we shall do so. Consider the power series expansions of $S_{t}{ }^{*} S_{t}$ and $S_{t} S_{t}^{*}$ :

$$
S_{t}^{*} S_{t}=I+t\left(A+A^{*}\right)+t^{2}\left(A^{2}+2 A^{*} A+A^{* 2}\right)+O\left(t^{3}\right)
$$

and

$$
S_{t} S_{t}^{*}=I+t\left(A+A^{*}\right)+t^{2}\left(A^{2}+2 A A^{*}+A^{* 2}\right)+O\left(t^{3}\right) .
$$

Since $\left(S_{t}\right)$ is centered, $S_{t}{ }^{*} S_{t}$ commutes with $S_{r} S_{r}^{*}$ for all nonnegative $t$ and $r$ [1, Lemma 2]. Consequently, the coefficients in the power series expansions of $S_{t}{ }^{*} S_{t}$ and $S_{t} S_{t}^{*}$ form an abelian set of operators. In particular, $A+A^{*}$ commutes with $A^{2}+2 A^{*} A+A^{* 2}$ and $A^{2}+2 A A^{*}+A^{* 2}$, and with their difference $2\left(A^{*} A-A A^{*}\right)$. That $A$ is normal follows from the next lemma.

Lemma. An operator $A$ on a Hilbert space $\mathscr{H}$ is normal if $A+A^{*}$ commutes with $A^{*} A-A A^{*}$.

Proof. Write $A=H+i K$ where $H=\left(A+A^{*}\right) / 2$ and $K=\left(A-A^{*}\right) / 2 i$. If $A+A^{*}$ commutes with $A^{*} A-A A^{*}$, then $H$ commutes with $H K-K H$. Kleinecke's result [2] on commutators implies that 0 is the only element in the spectrum $H K-K H$. Since $H$ and $K$ are Hermitian, this in turn implies that $H K-K H=0$ or equivalently that $A$ is normal.

Received by the editors February 17, 1984

1980 Mathematics Subject Classification. Primary 47D05, 47B20. 
Using the Theorem one can easily construct examples of semigroups for which the generator has nice algebraic properties not inherited by the semigroup itself. For example, if $A$ is a nonnormal centered operator [3] (that is, $\left\{A^{* n} A^{n}, A^{m} A^{* m}: m, n\right.$ nonnegative integers $\}$ is abelian), then $\left(e^{t A}\right)$ is nonnormal and, consequently, not centered. Similarly, if $A$ is a nonnormal, subnormal operator, then $\left(e^{t A}\right)$ is not centered.

We showed in the proof of the Theorem that $A$ is normal whenever the coefficients of the power series expansion of $S_{t}{ }^{*} S_{t}$ commute with those of $S_{t} S_{t}{ }^{*}$. These coefficients also arise in [4] where Lambert shows that $A$ is subnormal if each of the coefficients of $S_{t}^{*} S_{t}$ is nonnegative definite.

\section{REFERENCES}

1. M. Embry-Wardrop, The partially isometric factor of a semigroup, Indiana Univ. Math. J. 32 (1983), 893-901.

2. D. C. Kleinecke, On operator commutators, Proc. Amer. Math. Soc. 8 (1957), 535-536.

3. B. B. Morrell and P. S. Muhly, Centered operators, Studia Math. 51 (1974), 251-263.

4. A. Lambert, $\boldsymbol{A}$ characterization of subnormal operators, Glasgow Math. J. 25 (1984), 99-101.

Department of Mathematics, Central Michigan University, Mt. Pleasant, Michigan 48859 